\section{Bestrahlung plus BRAF-Inhibitor beim Melanom möglich?}

\section{Immer wieder wird von unerwarteten Nebenwirkungen berichtet, wenn mit BRAF-Inhibitoren behandelte Patienten mit Melanom bestrahlt werden. Dem wurde nun in einer retrospektiven multizentrischen Studie in elf europäischen Zentren nachgegangen.}

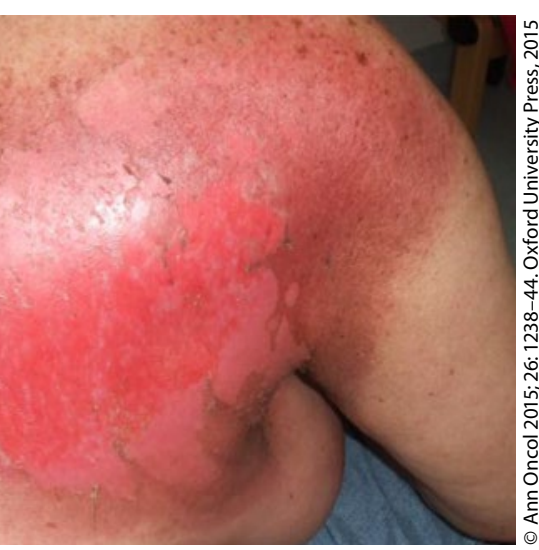

Akute Strahlendermatitis Grad 3 einer Patientin mit axillären Metastasen, die einen BRAF-Inhibitor erhielt und bestrahlt wurde

E in internationales Forscherteam hat Edie bei 161 Patienten mit Melanom aufgetretene akute und Spättoxizität analysiert. 70 dieser Patienten hatten insgesamt 86 Bestrahlungsserien parallel zur Therapie mit einem BRAF-Inhibitor erhalten. In Blutproben von 35 Patienten konnte auch in vitro ein individueller Test auf eine Radiosensibilisierung durchgeführt werden. Dazu wurde das Blut bestrahlt und mithilfe einer Fluoreszenz-in-situ-Hybribidisierung (FISH) auf Chromosomenbrüche hin untersucht.

Bei $57 \%$ der Bestrahlungen parallel zu einer BRAF-Inhibitor-Therapie trat eine akute oder Spättoxizität irgendeines Grades auf. Nebenwirkungen an der Haut waren sehr häufig, andere eher selten. So waren $36 \%$ der Patienten mit dieser Therapiekombination von einer akuten Strahlendermatitis $\geq$ Grad 2 betroffen, $12,8 \%$ von einer Proliferation follikulärer Zysten. Hörschäden waren mit $4 \%$ dagegen relativ selten, eine Dysphagie trat bei $2 \%$ der gleichzeitig mit einer BRAF-Inhibitor-Therapie bestrahlten Patienten auf. Nach einer
Ganzhirnbestrahlung lagen die Raten einer akuten Strahlendermatitis $\geq$ Grad 2 bei $8 \%$ bei bestrahlten Patienten ohne BRAF-Inhibitor-Therapie und bei $44 \%$ mit dieser Therapie. Dabei induzierte die gleichzeitige Therapie mit Vemurafenib häufiger eine Strahlendermatitis als die gleichzeitige Therapie mit Dabrafenib ( $40 \%$ vs. $26 \%$; $p=0,07$ ).

Die In-vitro-Untersuchungen bestätigten dies: Chromosomenbrüche bei Ex-vivo-Bestrahlung des Blutes von 35 Patienten traten bei Vemurafenib-Therapie und bei einer Sequenz von Vemurafenib gefolgt von Dabrafenib signifikant häufiger auf als ohne diese Therapien. Dagegen bestand kein signifikanter Unterschied in diesem In-vitro-Strahlensensitivitätsmodell zwischen Dabrafenib-Behandlung und keiner BRAF-Inhibitor-Therapie.

Fazit: Eine Bestrahlung bei laufender BRAF-Inhibitor-Therapie führt zu einem Anstieg der Toxizität, den die Autoren als akzeptabel bezeichnen. Dabei scheint Dabrafenib einen geringeren radiosensibilisierenden Effekt zu haben als Vemurafenib.

Friederike Klein

Hecht $M$ et al. Radiosensitization by BRAF inhibitor therapy-mechanism and frequency of toxicity in melanoma patients. Ann Oncol 2015; 26: 1238-44

\title{
Malignes Melanom der Bindehaut
}

Eine 78-jährige Frau mit Glaukom, Diabetes mellitus und Katarakt stellte sich routinemäßig zu einer augenärztlichen Untersuchung vor. Dabei stellte man eine rötlich-braune makulopapuläre Läsion am rechten Auge fest. Der Patientin war diese Veränderung seit etwa einem Jahr bewusst. Die Ausdehnung des Herdes hatte zu- und abgenommen, gelegentlich geblutet, war aber weder schmerzhaft noch hatte sie den Visus beeinträchtigt. Man führte eine sklerale Keratotomie mit anschließender Kryotherapie und Alkoholapplikation durch. Die histologische Untersuchung des Gewebes ergab ein malignes Melanom in Verbindung mit einer primär erworbenen Melanosis.

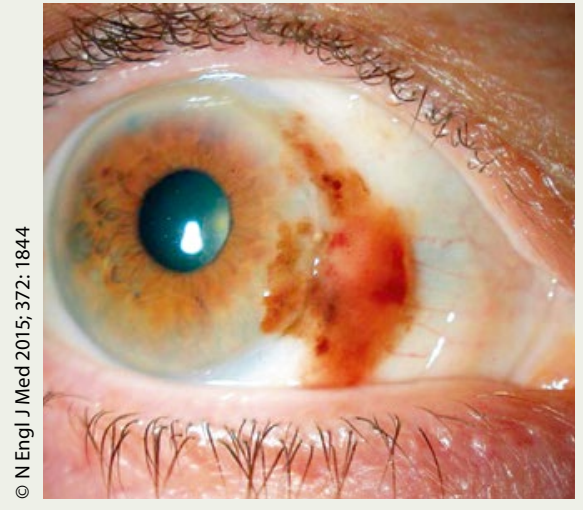

Rötlich-braune, gelegentlich blutende makulopapuläre Läsion am rechten Auge.
Konjunktivale Melanome können durch eine primär erworbene Melanosis entstehen und sind häufig mit regulären Metastasen und lokalen Rezidiven belastet. Die Patientin wurde mit Chemotherapie und topischer Anwendung von Mitomycin behandelt. Die Untersuchung der regulären Lymphknoten, ein Knochenszintigramm und ein Computertomogramm von Kopf, Hals, Brust und Abdomen ergaben keinen Hinweis auf eine Metastasierung. Auch 18 Monate nach Abschluss der Therapie zeigte sich kein Rezidiv.

Prof. Hermann S. FüeßI

Mclnnes CW \& Bellan L. Conjunctival melanoma. N Engl J Med 2015; 372: 1844 